\title{
Mutual understanding between Traditional Chinese Medicine and systems biology: gaps, challenges and opportunities
}

\section{Dong Xu}

Christopher S. Bond Life Sciences Center, Department of Computer Science, University of Missouri, Columbia 65211, MO, USA

E-mail: xudong@missouri.edu

\begin{abstract}
While Traditional Chinese Medicine (TCM) and systems biology have philosophical differences, both address human health using an integrative approach and both aim towards personalised medicine. The post-genomic era provides a unique opportunity for the two fields to understand and benefit from each other. In this review, the two approaches are compared, and various examples and hypotheses are provided to show how some practices in TCM might be interpreted by systems biology.
\end{abstract}

Keywords: TCM; traditional Chinese medicine; systems biology; CWM; conventional western medicine; personalised medicine; holism; herb; circadian rhythm; water structure; acupuncture points; ZHENG; Qi; qigong; body-mind relationship; gene expression; proteomics; metabolomics.

Reference to this paper should be made as follows: Xu, D. (2009) 'Mutual understanding between Traditional Chinese Medicine and systems biology: gaps, challenges and opportunities', Int. J. Functional Informatics and Personalised Medicine, Vol. 2, No. 3, pp.248-260.

Biographical notes: Dong $\mathrm{Xu}$ is James C. Dowell Professor and Chair of Computer Science Department, with appointments in the Christopher S. Bond Life Sciences Center and the Informatics Institute at the University of Missouri. He obtained his $\mathrm{PhD}$ from the University of Illinois, Urbana-Champaign in 1995 and did two-year postdoctoral work at the US National Cancer Institute. He was a Staff Scientist at Oak Ridge National Laboratory until 2003 before joining University of Missouri. His research includes protein structure prediction, high-throughput biological data analyses, in silico studies of plants, microbes, and cancers. He also has a long-time interest in traditional Chinese culture and medicine. He has published more than 150 papers in bioinformatics and computational biology.

\section{Introduction}

Although there are many debates on the values and usefulness of TCM, TCM is increasingly accepted and frequently practiced internationally beyond China. It has been widely accepted in East Asia for many generations. In the western world, TCM is also gaining ground as an effective alternative medical system. More importantly, TCM has brought much attention in the scientific research community worldwide. 
Figure 1 shows that the number of TCM-related publications in English-language journals has been increasing dramatically in the recent two decades. As of 1 October, 2009, there are 8629 PubMed-indexed papers containing the phrase 'Traditional Chinese Medicine', among which 3943 are in English (http://www.ncbi.nlm.nih.gov/pubmed/). More and more studies have demonstrated the effectiveness of TCM (e.g., Xu et al., 2007; Ceylan-Isik et al., 2008; Parekh et al., 2009; Lin and Huang, 2009, just to name a few). Through these studies, TCM is not only gaining public acceptance, but also becoming a real therapeutic option in western countries.

Figure 1 Annual number of PubMed-indexed papers in English containing the phrase 'Traditional Chinese Medicine' since 1990. The data was collected at PubMed (http://www.ncbi.nlm.nih.gov/pubmed/) on 1 October, 2009 (the number of publications in 2009 was incomplete for the year)

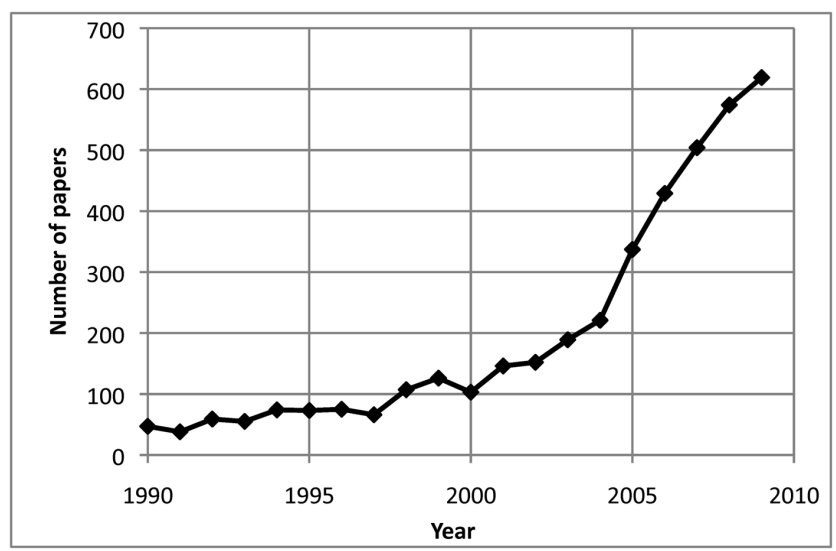

The differences between TCM and Conventional Western Medicine (CWM) are substantial and widely recognised. CWM is based on hard-core (well accepted) science, while TCM originated from oriental philosophy and culture, with a set of comprehensive, standalone theories and methodologies, which are often hard to be comprehended by Westerners. Although CWM is more broadly practiced and more effective in many cases, the value and impact of TCM in medical treatment are also ubiquitous. One may wonder how two dramatically different approaches can be both valuable and useful. To answer this question, it is important not to treat it as a logical problem, i.e., yes or no, right or wrong. CWM or TCM should not be used as the basis to judge its counterpart. Instead, their values should be assessed independently based on their contributions to the society and civilisation. To certain extent, the overlap between CWM and TCM reflects our ignorance on life and diseases. Using an analogy, if one draws 5 out of 54 poker cards twice independently, the odds of matching $>50 \%$ of cards are small; if one draws many more cards, the odds of matching will increase. In the same way, as we gain more insight from biomedical research, the differences and agreements between CWM and TCM will become more easily understood.

There has been extensive effort in bridging between CWM and TCM (Xutian et al., 2009). Due to historical reasons and its own methodologies, TCM was not institutionalised as effectively as CWM in broad training and systematic research. Furthermore, TCM often does not have standardised protocols that can be easily performed, repeated, and learned. Hence, the main effort in bridging between CWM and 
TCM has been made to apply scientific methods in understanding TCM, especially at the molecular levels (Parekh et al., 2009). In other words, TCM has been treated as a subject of science instead of a significant driver in pushing scientific developments. Nevertheless, there are many examples that TCM has provided interesting hypotheses in medical research, especially as useful leads in new drug development.

The advent of systems biology and personalised medicine provides a unique opportunity to bridge CWM and TCM. Some of the newly developed scientific approaches, such as viewing a disease as a systems problem and personalised treatments based on patients' conditions, echo TCM. Applying systems biology to study TCM can accelerate our understanding of TCM at the molecular levels. Meanwhile, it is possible to reformulate TCM theories, methods and practices so that they can provide a new driving force for modern health-related research and development. In this review paper, TCM and systems biology are compared. A number of examples are provided for suggesting hypotheses to interpret TCM theories and practices using systems biology and modern science in general. This review is not meant to be comprehensive on all related issues, but as an attempt to stimulate and share new ideas.

\section{Comparison between TCM and systems biology}

Many researchers have compared TCM with CWM (e.g., Griffiths, 1999). Nevertheless, with the advances in systems biology, the similarities and differences between TCM and CWM become more evident and insightful.

\subsection{Similarities between TCM and systems biology}

The gap between CWM and TCM is dramatically shrunk when employing systems biology as a research tool. There are four fundamental similarities between TCM and systems biology:

\section{Systematic view}

The integrative systems approaches of systems biology are in line with the holistic concept and practice of TCM. Driven by the success of human genome project, systems biology focuses on the systematic study of complex interactions in biological systems (Ideker et al., 2001; Sauer et al., 2007). In contrast to conventional approaches in life sciences, systems biology mainly targets discovering new emergent properties of a cell/organism viewed as an integrated and interacting network of genes, proteins and biochemical reactions (Palsson, 2006). TCM also views human body as an integrated system with networks, although the networks in TCM are not at the molecular levels and may not correspond to the CWM concepts. For example, the network of acupuncture points is an integrated system, but its mapping to CWM is still a subject of debates.

\section{Personalised medicine}

A major goal of systems biology is to advance predictive and personalised medicine, in particular, developing drugs on the basis of individual genetic differences. With $\$ 1000$ genome on the horizon, personalised medicine is actively pursued in biomedical research. Subtypes of diseases, together with their molecular biomarkers and specific treatments are studied. Pharmacogenomics applies genome-derived data to predict susceptibility to a 
disease or body's response to a drug, and as a result to prescribe medicine with maximum efficacies and minimum side effects. Although without a molecular basis, TCM also emphasises treatments based on personal conditions, including age, gender, personality, weight, concomitant diseases, concomitant medicine, social factors, etc. In TCM, a disease is often categorised into subtypes for different treatments. For example, flu can be classified based on its ZHENG, a TCM abstraction of the symptom profiles for a disease, to customise herb formula. A recent study suggests that different ZHENGs (e.g., Hot ZHENG and Cold ZHENG) may be related to particular biomolecular networks (Li et al., 2007). Furthermore, one disease may be attributed to multiple roots/causes and be treated by multiple formulae of herbs, while multiple diseases might share the same root/cause and be treated by the same formula. Additionally, the treatments in TCM often tie with personalities of patients. Modern biomedical research also starts to recognise the connections between personality and disease/treatment, e.g., the relationship between type A personality and heart disease (Friedman, 1996), while the genetic basis of personality is being explored (Rebollo and Boomsma, 2006).

\section{Based on observations and experimentations}

Although systems biology and TCM have different methodologies, it is important to recognise that both of them rely on observations and experimentations. Observation and experimentation also separate TCM from superstition. According to Chinese legend, Shen Nong, a ruler of China who lived about 5000 years ago, tasted hundreds of herbs to test their medical effects. The therapeutic methods and herb medicines in TCM have been the results of countless such ad hoc experimental trials over many generations, which built a strong basis for TCM. In this regard, methods in TCM are usually objective and repeatable/predictable in terms of outcomes. Hence, it is possible to automate some TCM practices. For example, computerised tongue examination systems were successfully developed to mimic the TCM tongue diagnosis (Chiu, 2000).

\section{Theory driven}

In contrast to most other alternative medicines, TCM has systematic theoretical foundations ( $\mathrm{Lu}$ et al., 2004). Although there are major philosophical differences between CWM and TCM, both are guided by theories. Both systems biology and TCM have extensive theories on how a biological system functions with regulation among different components, interactions with its environments, abnormal states (diseases), and corrections (treatments) of abnormal states.

\subsection{Difference between TCM and systems biology}

Although there are many similarities between TCM and systems biology, it is also important to recognise their fundamental differences:

\section{Reductionism vs. holism}

Although systems biology focuses on emerging properties of systems and is much more holistic than conventional biomedical research, it is still a continuation of science driven mainly by reductive approaches. Systems biology builds upon the necessity of dividing a system into separate parts; in particular, a biological system is still reduced into a set of molecules (DNA, proteins, etc.), which form multiple levels of structures, including cells, tissues and organs. In contrast, the holistic approaches of TCM do not 
rely on understanding of molecular mechanisms. Some of the concepts in TCM, such as Qi and Yin-Yang, may not be able to map into scientific terms, at least at this stage (Wiseman, 2006).

\section{Bottom up vs. top-down}

With the comparison of reductionism vs. holism between systems biology and TCM, systems biology mainly uses bottom-up methodologies while TCM is primarily based on top-down approaches. A goal of systems biology is to integrate the molecular mechanisms to understand the properties and behaviour of high-level structures, and as a result to develop predictive models. There are many component variables used in describing a system, such as gene copy number, protein expression levels, abundance of metabolites, etc., in systems biology. In contract, TCM uses many systems-level characteristics linking to the disease. Some of these characteristics are shared with CWM, such as body temperature and heart rate, but many more are not emphasised in CWM, such as facial colour, smell of mouth, morphological features, etc.

\section{External observation vs. internal observation}

CWM and systems biology emphasise data while TCM relies more on experiences. Systems biology treats human body as an object; it measures physical existence in a human body as objective as possible and in a similar way as studying an inorganic molecule through external observation. The collected data are typically repeatable. Systems biology handles gene networks, cells, etc., as the tangible engineered systems. The wonderful design principles of biological systems are generally attributed to evolution in the scientific community, while some believe such 'intelligent design' came from divinity (Pennock, 2003). On the other hand, TCM handles 'subject-object problem' or 'body-mind problem' quite differently from CWM. It often assumes that observer (as a subject) and its human body (as an object) are indivisible. Hence, many studies rely on internal observation instead of physical measurements. For example, it was suggested that the acupuncture points were discovered from Qigong (http://en.wikipedia. org/wiki/Qigong), an internal Chinese meditative practice and hence, it might not have physical entities, although some physical effects might be detected (Pearson et al., 2007). The system properties are often attributed to Qi (energy flow; http://en.wikipedia. org/wiki/Qi) without assuming physical basis or supernatural intervention. For the treatment, TCM also emphasises the emotion and feeling of patients.

\section{Crisp vs. fuzzy}

CWM is often quantitative with high precision. Recent medical advancements have been remarkable in this regard. For example, microsurgeries can achieve 1-mm precision anastomosis of small blood vessels and nerves for transferring tissue from one part of the body to another. Systems biology can deliver many data with digital quality, such as Single-Nucleotide Polymorphism (SNP) at the genome scales. On the other hand, TCM uses many concepts that are judged as 'fuzzy' by science. Due to the fuzzy features of many TCM concepts, it is challenging to map most of them into scientific terms (Wiseman, 2006). In contrast to CWM, which generally has well-established clinical trials involving rigorous statistical analyses for medical treatments, TCM does not have such a quantitative system and many treatments are based on experience in an ad hoc fashion. Although clinical trials of TCM are usually feasible and necessary for testing the efficacy of TCM (Ernst, 2006), the quantitative approaches of CWM may not always be 
suitable for evaluating TCM, which aims to correct maladjustments and restore the self-regulatory ability of the body instead of antagonising specific pathogenetic targets (Jiang, 2005). Some evaluation methods for 'evidence-based medicine' may require modifications to better assess the medical value of TCM (Shea, 2006).

5 Offence vs. defence in disease development and treatment

A disease often has an offence side (disease agents, e.g., bacteria, viruses, cancer cells, etc.) and a defence side (e.g., immune system). CWM usually targets the offence side, for example to develop drug compounds that specifically bind molecules (e.g., protein receptors) of these disease agents and ultimately kill the agents. The advance in systems biology provides some new understanding on how the disease agents work at the systems levels (Laubenbacher et al., 2009). On the other hand, TCM focuses more on the defence side. It attributes many diseases to imbalance or weakness of human system. For example, flu viruses are ubiquitous in the environment. However, whether one gets flu may not depend only on access to these viruses. As English language of 'catching a cold' for flu, TCM often regards the weakness of defence system due to such environmental factors (e.g., temperature and wind), emotion, stress, etc., as causes of diseases. The goal of TCM is to treat the diseases based on these causes rather than to simply alleviate their symptoms. Hence, herb formulas are often designed to repair/boost the disease defense system. Furthermore, TCM stresses the prevention of diseases by strengthening the disease defence systems through developing and maintaining healthy lifestyle, where TCM has a very rich culture. Having said that, it is noted that CWM also started to promote healthy lifestyle in disease prevention, as ultimate cure for certain diseases such as cancers is becoming less hopeful to come in the near future (Demark-Wahnefried et al., 2008).

\section{Examples of reconciliation}

Active biomedical research, especially systems biology on studying TCM in recent years has helped bridge the gap between CWM and TCM. In this section, five examples are given to show how ongoing research helped reconcile between CWM and TCM. A few hypotheses are provided to show that some TCM practices might be understood from scientific perspectives. Many other systems biology studies in TCM are not illustrated in this review, such as building the genome-phenome relationships (Li-Ling, 2003), using genetic imaging methods and microarray imaging for personalised medicine in TCM (Chen et al., 2009), identifying network biomarkers for TCM syndrome and herb formula (Li, 2009), and applying systems toxicology to guide the safety and quality of Chinese herbal medicine (Wang et al., 2009).

\subsection{Herb composition and preparation}

Herbal medicine is not unique in TCM. Many alternative medicines apply herbs, whose medicinal effects were discovered through life experiences. Some drugs used in CWM were also derived from nature products in plants. For example, aspirin (acetylsalicylic acid) is a derivative of salicylic acid found in some plants, such as willow, which was used for reducing pain and fever even back in ancient Egypt and Greece. In a way, the usefulness of plant in medicine is not surprising. Plants developed much stronger 
capacities to resist pathogens and environmental stresses than animals, as plants cannot move like animals under these conditions. The compounds related to biotic and abiotic stress responses in plants are plentiful, and they can help animals (including human) in a similar way. As an example, salicylic acid is a naturally occurring compound in plants to stimulate the plants' internal defence pathways (Crampton et al., 2009). In human its derivative aspirin can exert modulatory effects on the innate and adaptive immune systems through the COX-2 gene (Gilroy, 2005).

Unlike natural compounds used in CWM or other alternative medicines, most traditional herb formulas consist of a combination of several plants. The combination is often aimed towards achieving the maximum therapeutic efficacy and the minimum side effects by facilitating synergistic actions towards multiple targets. In some cases, the therapeutic efficacy may only be attained by the combination in a similar way as genetic synthetic lethality, where two separate viable mutations combined in a double mutant result in lethality (Tong et al., 2004). Biomedical studies also support the effectiveness of TCM's multicomponent, multitarget approach (Sucher, 2006). In almost all cases, bioactive components in herb formulas are unknown. One may assume that only a few compounds are responsible for the therapeutic or toxic effects, but their screening and separation are challenging (Liu et al., 2008). Recent studies applied metabolite profiling to identify the natural compounds in large scales (Dan et al., 2007; Xie et al., 2008), which will provide a systems-level understanding on herbs. Metabolomic studies for Chinese herbs will shed some light on how various compounds work in synergy for medical treatment (Wang et al., 2005).

Herbs used in TCM are often prepared in particular protocols. In many cases, the herbs are boiled for certain period of time. This is understandable, as the elevated temperature will allow the herbs to break down into small molecules and help certain chemical reactions among these molecules. On the other hand, some protocols require quenching by addition of ice water or snow water. In the well-known pharmacopoeia, the Compendium of Materia Medica (Bencao Gangmu) by Dr. Li Shizhen (1518 A.D. to 1594 A.D.) in Ming Dynasty, water is classified into different types for drinking and preparing herbs, such as rain water, dew water, frost water, snow water, ice water, river water, well water, hot spring, etc. Apart from different minerals in various water types, modern science finds the structures of liquid water can be different. Sometimes, thermally disordered, ice-like cluster structures are in water (Mantz et al., 2006). Other studies identified complexes between water molecules and small, oxygen-containing inorganic oxidants in the atmosphere such as $\mathrm{O}_{3}$ and $\mathrm{H}_{2} \mathrm{O}_{2}$ (Sennikov et al., 2005). Although TCM never used such molecular mechanisms as a basis, these mechanisms could lead to various effects of herbs from different sources of water. To my knowledge, the biomedical effects of different water types have not been explored at the molecular levers, and this could be a subject for future studies.

\subsection{Transcriptomic and proteomic response to TCM}

Transcriptomics and proteomics, as important systems biology technologies have been used in studying TCM. Both technologies can be applied to simultaneously study the expressions, functions, and dynamics of all genes in a genome. They have been applied in studying TCM pharmacological effects and mechanisms in terms of mRNA/protein expression (Cho, 2007; Kang, 2008). DNA microarrays showed that treatment with Huanglian (Coptidis rhizoma, a widely used TCM herb), dramatically increased the gene 
expression of interferon-beta (IFN-beta) and tumour necrosis factor-alpha in MCF-7 cells, which suggests Huanglian's antiproliferative effect (Kang et al., 2005). Microarray technology was also applied to study the effect of VI-28 (a TCM formula) on gene expression in human lymphocytes and it was found that the VI-28 treatment induced expression of several proinflammatory cytokines/chemokines in both peripheral blood mononuclear cells and spleen cells (Pan-Hammarström et al., 2006). Another microarray gene expression study indicates that TCM herbal formula San-Huang-Xie-Xin-Tang and its components displayed a unique anti-proliferation pattern via p53 signalling and DNA damage signalling pathways in HepG2 cells (Cheng et al., 2008b). A proteomic analysis was conducted on HepG2 cells treated by Rhizoma Paridis (Zao Xiu), a TCM herb to treat many diseases including tumour (Cheng et al., 2008a). The study found that a number of proteins associated with tumour initiation, promotion, and progression were differentially expressed under the treatment, which shed some light on the mechanism of the herb's anti-tumour effects. Another proteomic study indicates Tubeimoside-1 extracted from TCM anticancer Bolbostemma paniculatum Franquet, exhibited cytotoxicity in HeLa cells through both mitochondrial dysfunction and ER stress cell death pathways (Xu et al., 2009).

\subsection{Biological clock: body cycle and timing of treatment}

TCM emphasises the circadian rhythm of illness and applies it to schedule therapies (chronotherapy) such as acupuncture during appropriate hours of the day. TCM also illustrates organ-specific biorhythms for lungs, stomach, heart, kidneys, liver, etc., and modern medical research has found similar patterns (Samuels, 2000). More and more systems biology studies are uncovering the relationships between the circadian rhythm and human health at molecular levels. A recent metabolomics and bioinformatics study (Wang et al., 2008) shows systematic circadian variations of metabolites in urine from healthy men at different time of a day. Circadian transcriptional network models based on gene-expression and promoter-element analyses suggested some regulatory mechanism of the circadian rhythm (e.g., Hayes et al., 2005). Another study also shows that the biological clocks vary in a tissue-specific manner, supporting the concept of organ-specific biorhythms in TCM (Ko and Takahashi, 2006).

\subsection{Relationships between body and mind}

TCM links emotions and physical health closely and regards psychological well being an overarching factor in health and treatment (Ma, 2005). The connections between psychological conditions and diseases are not unique in TCM. Such connections are illustrated well through the relationship between psychological stresses and immune system suppressions (Reiche et al., 2004). A unique aspect of TCM is to associate each emotion (sadness, anger, worry, fear, etc.) with a particular organ (Ots, 1990). For example, anger is connected with liver, i.e., anger can negatively affect the liver and physiological problems of liver can trigger irritability. A possible reason for the connection is that the liver plays an important role in blood flow throughout the body and some biomolecules from liver (e.g., hormones) may affect the emotion, although it is worth pointing out that the liver in TCM does not fully correspond to the liver in CWM (Wiseman, 2006). TCM is well-seeded in Qigong, which can help build both physiological and psychological balances simultaneously thorough meditation, 
breath control, and slow body motion (Xu, 1994; Kjos and Etnier, 2006). Systems biology methods have been applied to study the body-mind relationships, especially in Qigong. For example, a study suggests that Qigong practice may regulate immunity, metabolic rate, and cell death at the transcriptional level (Li et al., 2005).

\subsection{Systems-level modelling}

TCM has applied many sophisticated theories such as Yin-Yang, Wu-Xing (Five-Element theory), and Jing-Mai (Meridians) to study human as systems, whose descriptions are dramatically different from the systems in which systems biology adopts. While there have been efforts to bridge the two, an intriguing question is whether scientific/engineering techniques can help use these TCM theories directly, i.e., to model 'authentic' TCM without a molecular basis. For example, it may be possible to apply bona fide TCM foundations with modern computational and modelling techniques in deriving models so that TCM becomes more operational and predictive for general practitioners, although these models may not be needed for a handful of TCM masters. Along this line, Kang et al. (2008) applied the entropy theory to model Qi in TCM. Ding and Wang (2008) developed a Wu-Xing dynamic model for TCM to study the transition between the healthy state and the sub-healthy state. Chen and Zhang (2008) formulated an equilibrium/non-equilibrium computational model of Yin-Yang and Wu-Xing based on the bipolar set theory. They also performed computer simulations, which showed that this new approach could provide diagnostic decision support in TCM. The same model can be extended to molecular levels to explain the behaviour of Toll-like receptors in the innate immune system using a network structure (Jaeger et al., 2009).

\section{Conclusion and discussion}

Systems biology is providing a historical golden opportunity for a renaissance of TCM and a bridge between TCM and CWM. This unique opportunity can help TCM and CWM to benefit from each other. On one hand, systems biology provides a tool for science to understand TCM from molecular levels. In this regards, future studies, especially with next-generation sequencing methods will provide more insight on the genomic sequences of herbs, molecular responses (protein, non-coding RNA, etc.) of TCM, etc. On the other hand, TCM may suggest useful hypotheses in leads of drugs, personalised medicine, and healthy lifestyle, all of which can be important supplements to CWM.

There is still a long way to go to reconcile between TCM and CWM due to their fundamental philosophical differences. Although systems biology has identified various molecular phenomena associated with TCM, it does not mean the phenomena represent a full picture or a molecular basis for TCM. Many of the concepts in TCM do not focus on the physical substances but rather on the emerging properties of the human body-mind system directly. These emerging properties come from physical substances, and hence systems biology can trace them in various molecular aspects including gene/protein expression and metabolites, as discussed in this review. Meanwhile, they also have macroscopic effects, e.g., acupuncture points can have different temperature or electrical properties (Pearson et al., 2007). Nevertheless, all these molecular/ physical measurements are 'components' and 'reflections' (instead of the essence) of the 
emerging properties. In this regard, TCM and CWM are built on different hypotheses like Euclidean geometry and non-Euclidean geometry. It requires new frameworks to reconcile the two. It is destructive to judge the value of one merely from another or label TCM as pseudo-science, especially since biomedical research is discovering new things on daily basis and some knowledge in today's biology textbook may be proven to be incorrect in the future as it has been.

TCM is at a historical turning point. At the positive side, systems biology starts to demonstrate the values of TCM from scientific perspectives. The related research may be further empowered by the remarkable economic growth in China. Many researchers outside TCM, including myself, are eager to help connect TCM with modern science. On the flip side, TCM had few novel developments during the past two centuries and it has been marginalised as CWM has been continuously making advances during the same period. TCM has not been adapted to modern society well. Other than the limitations in theories and practices, TCM did not have a good infrastructure for the developments. Traditionally, TCM was mainly conveyed from teacher to student in an ad hoc fashion, often within a family. It lacks of a general education and research infrastructure in which science has been developed, such as universities, conferences, funding agencies, journals, open access, public databases, standardisation, etc. It is promising to see that the TCM research infrastructure is being developed. Database and data mining in TCM have been conducted (Feng et al., 2006; Chen et al., 2007; You et al., 2009). Nevertheless, there are many aspects of CWM that TCM can further learn from. When TCM reinvents itself in theories, practices, and infrastructure with the advent of systems biology, it will benefit a much larger world population for many generations to come.

\section{Acknowledgements}

I would like to dedicate this review paper to Mr. Jia Titao, whose centenary anniversary is this year (2009) and Mr. Wang Yonghui, who passed away last year. Both teachers taught me traditional Chinese philosophy and its practice. I also thank Hao-Teng Chang, Jingxi Chu, Leo Lee, Shao Li and Yanqing Zhang for critical readings and helpful comments of this paper.

\section{References}

Ceylan-Isik, A.F., Fliethman, R.M., Wold, L.E. and Ren, J. (2008) 'Herbal and traditional Chinese medicine for the treatment of cardiovascular complications in diabetes mellitus', Curr. Diabetes Rev., Vol. 4, pp.320-328.

Chen, H., Mao, Y., Zheng, X., Cui, M., Feng, Y., Deng, S., Yin, A., Zhou, C., Tang, J., Jiang, X. and Wu, Z. (2007) 'Towards semantic e-Science for traditional Chinese medicine', BMC Bioinformatics, Vol. 8, Suppl. 3, p.S6.

Chen, S.S. and Zhang, W.R. (2008) 'One Yin-Yang Wu-Xing model of TCM', Journal of Acupuncture and Tuina Science, Vol. 6, pp.266-268.

Chen, S.S., Song, Q.F. and Wang, Y.P. (2009) 'Genomic imaging: a modern environment for TCM research', Proceedings of the First International Joint Conference on Bioinformatics, Systems Biology and Intelligent Computing (IJCBS09), 2-5 August, Shanghai, China, pp.371-376. 
Cheng, W.Y., Wu, S.L., Hsiang, C.Y., Li, C.C., Lai, T.Y., Lo, H.Y., Shen, W.S., Lee, C.H., Chen, J.C., Wu, H.C. and Ho, T.Y. (2008b) 'Relationship between San-Huang-Xie-Xin-Tang and its herbal components on the gene expression profiles in HepG2 cells', Am. J. Chin. Med., Vol. 36, pp.783-797.

Cheng, Z.X., Liu, B.R., Qian, X.P., Ding, Y.T., Hu, W.J., Sun, J. and Yu, L.X. (2008a) 'Proteomic analysis of anti-tumor effects by Rhizoma Paridis total saponin treatment in HepG2 cells', J. Ethnopharmacol., Vol. 120, pp.129-137.

Chiu, C.C. (2000) 'A novel approach based on computerized image analysis for traditional Chinese medical diagnosis of the tongue', Comput. Methods Programs Biomed., Vol. 61, pp.77-89.

Cho, W.C. (2007) 'Application of proteomics in Chinese medicine research', Am. J. Chin. Med., Vol. 35, pp.911-922.

Crampton, B.G., Hein, I. and Berger, D.K. (2009) 'Salicylic acid confers resistance to a biotrophic rust pathogen, Puccinia substriata, in pearl millet (Pennisetum glaucum)', Mol. Plant Pathol., Vol. 10, pp.291-304.

Dan, M., Gao, X.F., Xie, G.X., Liu, Z., Zhao, A.H. and Jia, W. (2007) 'Application of metabolomics in research of plant metabolites', Zhongguo Zhong Yao Za Zhi, Vol. 32, pp.2337-2341.

Demark-Wahnefried, W., Rock, C.L., Patrick, K. and Byers, T. (2008) 'Lifestyle interventions to reduce cancer risk and improve outcomes', Am. Fam. Physician, Vol. 77, pp.1573-1578.

Ding, H. and Wang, T. (2008) 'Mathematical analysis of Yin-Yang Wu-Xing model in TCM', Journal of Acupuncture and Tuina Science, Vol. 6, pp.269-270.

Ernst, E. (2006) 'Methodological aspects of Traditional Chinese Medicine (TCM)', Ann. Acad. Med. Singapore, Vol. 35, pp.773-774.

Feng, Y., Wu, Z., Zhou, X., Zhou, Z. and Fan, W. (2006) 'Knowledge discovery in traditional Chinese medicine: state of the art and perspectives', Artif. Intell. Med., Vol. 38, pp.219-236.

Friedman, M. (1996) Type A Behavior: Its Diagnosis and Treatment, Plenum Press (Kluwer Academic Press), New York.

Gilroy, D.W. (2005) 'The role of aspirin-triggered lipoxins in the mechanism of action of aspirin', Prostaglandins Leukot Essent. Fatty Acids, Vol. 73, pp.203-210.

Griffiths, V. (1999) 'Eastern and Western paradigms: the holistic nature of traditional Chinese medicine', Aust. J. Holist Nurs., Vol. 6, pp.35-38.

Hayes, K.R., Baggs, J.E. and Hogenesch, J.B. (2005) 'Circadian clocks are seeing the systems biology light', Genome Biol., Vol. 6, p.219.

Ideker, T., Galitski, T. and Hood, L. (2001) 'A new approach to decoding life: systems biology', Annu. Rev. Genomics Hum. Genet., Vol. 2, pp.343-372.

Jaeger, S., Chen, S.S. and Zhang, W.R. (2009) 'TCM in innate immunity: a YinYang-N-element hypothesis of the innate immunological system', Proceedings of the First International Joint Conference on Bioinformatics, Systems Biology and Intelligent Computing (IJCBS09), 2-5 August, Shanghai, China, pp.397-401.

Jiang, W.Y. (2005) 'Therapeutic wisdom in traditional Chinese medicine: a perspective from modern science', Trends Pharmacol. Sci., Vol. 26, pp.558-363.

Kang, G.L., Li, S. and Zhang, J.F. (2008) 'Entropy-based model for interpreting life systems in traditional Chinese medicine', Evid Based Complement Alternat. Med., Vol. 5, pp.273-279.

Kang, J.X., Liu, J., Wang, J., He, C. and Li, F.P. (2005) 'The extract of Huanglian, a medicinal herb, induces cell growth arrest and apoptosis by upregulation of interferon-beta and TNF-alpha in human breast cancer cells', Carcinogenesis, Vol. 26, pp.1934-1939.

Kang, Y.J. (2008) 'Herbogenomics: from traditional Chinese medicine to novel therapeutics', Exp. Biol. Med. (Maywood), Vol. 233, pp.1059-1065.

Kjos, V. and Etnier, J.L. (2006) 'Pilot study comparing physical and psychological responses in medical qigong and walking', J. Aging Phys. Act., Vol. 14, pp.241-253. 
Ko, C.H. and Takahashi, J.S. (2006) 'Molecular components of the mammalian circadian clock', Hum. Mol. Genet., Vol. 15, pp.R271-R277.

Laubenbacher, R., Hower, V., Jarrah, A., Torti, S.V., Shulaev, V., Mendes, P., Torti, F.M. and Akman, S. (2009) 'A systems biology view of cancer', Biochim. Biophys. Acta., Vol. 1796, pp.129-139.

Li, Q.Z., Li, P., Garcia, G.E., Johnson, R.J. and Feng, L. (2005) 'Genomic profiling of neutrophil transcripts in Asian Qigong practitioners: a pilot study in gene regulation by mind-body interaction', J. Altern. Complement Med., Vol. 11, pp.29-39.

Li, S. (2009) 'Network systems underlying traditional Chinese medicine syndrome and herb formula', Current Bioinformatics, Vol. 4, pp.188-196.

Li, S., Zhang, Z.Q., Wu, L.J., Zhang, X.G., Li, Y.D. and Wang, Y.Y. (2007) 'Understanding ZHENG in traditional Chinese medicine in the context of neuro-endocrine-immune network', IET Syst. Biol., Vol. 1, pp.51-60.

Li-Ling, J. (2003) 'Human Phenome based on traditional Chinese medicine - a solution to congenital syndromology', Am. J. Chin. Med., Vol. 31, pp.991-1000.

Lin, J. and Huang, W.W. (2009) 'A systematic review of treating Helicobacter pylori infection with traditional Chinese medicine', World J. Gastroenterol., Vol. 15, pp.4715-4719.

Liu, S., Yi, L.Z. and Liang, Y.Z. (2008) 'Traditional Chinese medicine and separation science', J. Sep. Sci., Vol. 31, pp.2113-2137.

Lu, A.P., Jia, H.W., Xiao, C. and Lu, Q.P. (2004) 'Theory of traditional Chinese medicine and therapeutic method of diseases', World J. Gastroenterol., Vol. 10, pp.1854-1856.

Ma, S.S. (2005) 'The I Ching and the psyche-body connection', J. Anal. Psychol., Vol. 50, pp.237-250.

Mantz, Y.A., Chen, B. and Martyna, G.J. (2006) 'Structural correlations and motifs in liquid water at selected temperatures: ab initio and empirical model predictions', J. Phys. Chem. B, Vol. 110, pp.3540-3554.

Ots, T. (1990) 'The angry liver, the anxious heart and the melancholy spleen. The phenomenology of perceptions in Chinese culture', Cult. Med. Psychiatry, Vol. 14, pp.21-58.

Palsson, B. (2006) Systems Biology - Properties of Reconstructed Networks, Cambridge University Press, Cambridge, UK.

Pan-Hammarström, Q., Wen, S. and Hammarström, L. (2006) 'Cytokine gene expression profiles in human lymphocytes induced by a formula of traditional Chinese medicine, vigconic VI-28', J. Interferon Cytokine Res., Vol. 26, pp.628-636.

Parekh, H.S., Liu, G. and Wei, M.Q. (2009) 'A new dawn for the use of traditional Chinese medicine in cancer therapy', Mol. Cancer, Vol. 8, p.21.

Pearson, S., Colbert, A.P., McNames, J., Baumgartner, M. and Hammerschlag, R. (2007) 'Electrical skin impedance at acupuncture points', J. Altern. Complement Med., Vol. 13, pp.409-418.

Pennock, R.T. (2003) 'Creationism and intelligent design', Annu. Rev. Genomics Hum. Genet., Vol. 4, pp.143-163.

Rebollo, I. and Boomsma, D.I. (2006) 'Genetic analysis of anger: genetic dominance or competitive sibling interaction', Behav. Genet., Vol. 36, pp.216-228.

Reiche, E.M., Nunes, S.O. and Morimoto, H.K. (2004) 'Stress, depression, the immune system, and cancer', Lancet Oncol., Vol. 5, pp.617-625.

Samuels, N. (2000) 'Chronotherapy in traditional Chinese medicine', Am. J. Chin. Med., Vol. 28, pp.419-423.

Sauer, U., Heinemann, M. and Zamboni, N. (2007) 'Genetics. Getting closer to the whole picture', Science, Vol. 316, pp.550-551.

Sennikov, P.G., Ignatov, S.K. and Schrems, O. (2005) 'Complexes and clusters of water relevant to atmospheric chemistry: $\mathrm{H}_{2} \mathrm{O}$ complexes with oxidants', Chemphyschem., Vol. 6, pp.392-412. 
Shea, J.L. (2006) 'Applying evidence-based medicine to traditional Chinese medicine: debate and strategy', J. Altern. Complement Med., Vol. 12, pp.255-263.

Sucher, N.J. (2006) 'Insights from molecular investigations of traditional Chinese herbal stroke medicines: implications for neuroprotective epilepsy therapy’, Epilepsy Behav., Vol. 8, pp.350-362.

Tong, A.H., Lesage, G., Bader, G.D., Ding, H., Xu, H., Xin, X., Young, J., Berriz, G.F., Brost, R.L., Chang, M., Chen, Y., Cheng, X., Chua, G., Friesen, H., Goldberg, D.S., Haynes, J., Humphries, C., He, G., Hussein, S., Ke, L., Krogan, N., Li, Z., Levinson, J.N., Lu, H., Ménard, P., Munyana, C., Parsons, A.B., Ryan, O., Tonikian, R., Roberts, T., Sdicu, A.M., Shapiro, J., Sheikh, B., Suter, B., Wong, S.L., Zhang, L.V., Zhu, H., Burd, C.G., Munro, S., Sander, C., Rine, J., Greenblatt, J., Peter, M., Bretscher, A., Bell, G., Roth, F.P., Brown, G.W., Andrews, B., Bussey, H. and Boone, C. (2004) 'Global mapping of the yeast genetic interaction network', Science, Vol. 303, pp.808-813.

Wang, J., van der Heijden, R., Spruit, S., Hankermeier, T., Chan, K., van der Greef, J., Xu, G. and Wang, M. (2009) 'Quality and safety of Chinese herbal medicines guided by a systems biology perspective', J. Ethnopharmacol., Vol. 126, pp.31-41.

Wang, M., Lamers, R.J., Korthout, H.A., van Nesselrooij, J.H., Witkamp, R.F., van der Heijden, R., Voshol, P.J., Havekes, L.M., Verpoorte, R. and van der Greef, J. (2005) 'Metabolomics in the context of systems biology: bridging traditional Chinese medicine and molecular pharmacology', Phytother. Res., Vol. 19, pp.173-182.

Wang, X., Lv, H., Zhang, G., Sun, W., Zhou, D., Jiao, G. and Yu, Y. (2008) 'Development and validation of a ultra performance LC-ESI/MS method for analysis of metabolic phenotypes of healthy men in day and night urine samples', J. Sep. Sci., Vol. 31, pp.2994-3001.

Wiseman, N. (2006) 'Concerning the use of Western medical terms to represent traditional Chinese medical concepts', Chin. J. Integr. Med., Vol. 12, pp.225-228.

Xie, G., Plumb, R., Su, M., Xu, Z., Zhao, A., Qiu, M., Long, X., Liu, Z. and Jia, W. (2008) 'Ultra-performance LC/TOF MS analysis of medicinal Panax herbs for metabolomic research', J. Sep. Sci., Vol. 31, pp.1015-1026.

Xu, L., Lao, L.X., Ge, A., Yu, S., Li, J. and Mansky, P.J. (2007) 'Chinese herbal medicine for cancer pain', Integr. Cancer Ther., Vol. 6, pp.208-234.

$\mathrm{Xu}$, S.H. (1994) 'Psychophysiological reactions associated with qigong therapy', Chin. Med. J. (Engl), Vol. 107, pp.230-233.

Xu, Y., Chiu, J.F., He, Q.Y. and Chen, F. (2009) 'Tubeimoside-1 exerts cytotoxicity in HeLa cells through mitochondrial dysfunction and endoplasmic reticulum stress pathways', J. Proteome Res., Vol. 8, pp.1585-1593.

Xutian, S., Zhang, J. and Louise, W. (2009) 'New exploration and understanding of traditional Chinese medicine', Am. J. Chin. Med., Vol. 37, pp.411-426.

You, M., Ge, L., Li, G-Z., Xu, L. and Huang, S. (2009) 'A TCM platform for masters' experience sharing', Proceedings of the First International Joint Conference on Bioinformatics, Systems Biology and Intelligent Computing (IJCBS09), 2-5 August, Shanghai, China, pp.388-391. 